\title{
On the 3-form formulation of axion potentials from D-brane instantons
}

\author{
Eduardo García-Valdecasas ${ }^{a, b}$ and Angel Uranga ${ }^{a}$ \\ ${ }^{a}$ Instituto de Física Teórica UAM-CSIC, \\ C/Nicolás Cabrera 13-15, Campus de Cantoblanco, 28049 Madrid, Spain \\ ${ }^{b}$ Departamento de Fúsica Teórica, Universidad Autónoma de Madrid, \\ Campus de Cantoblanco, 28049 Madrid, Spain \\ E-mail: eduardo.garcia.valdecasas@gmail.com, angel.uranga@uam.es
}

ABSTRACT: The study of axion models and quantum corrections to their potential has experienced great progress by phrasing the axion potential in terms of a 3 -form field eating up the 2-form field dual to the axion. Such reformulation of the axion potential has been described for axion monodromy models and for axion potentials from non-perturbative gauge dynamics. In this paper we propose a 3 -form description of the axion potentials from non-gauge D-brane instantons. Interestingly, the required 3-form field does not arise in the underlying geometry, but rather shows up in the KK compactification in the generalized geometry obtained when the backreaction of the D-brane instanton is taken into account.

KEYWORDS: D-branes, Flux compactifications, Gauge-gravity correspondence

ARXIV EPRINT: 1605.08092 


\section{Contents}

1 Introduction and main results 1

2 Review of 3-forms and monodromy. 2

3 3-forms from D-brane instanton backreaction 4

3.1 The puzzle 4

3.2 D-brane instanton backreaction 5

3.3 The 3-form and its coupling 6

$\begin{array}{ll}3.4 & \text { Some toroidal examples }\end{array}$

3.5 Generalization 8

4 Gauge non-perturbative effects $\quad 9$

5 Conclusions $\quad 10$

\section{Introduction and main results}

Axions have become an essential template to describe physics of scalar fields whose potential enjoys special protection properties due to an underlying symmetry principle. Naively, the symmetry corresponds to the perturbative global symmetry shifting the value of the scalar field, which is violated by non-perturbative effects, as originally proposed for the QCD axion [1]. However, it has recently become clear that the most fundamental symmetry structure is that of the dual 2-form. Contributions to the axion potential which spoil the shift symmetry must arise from the existence of a 3-form which eats up the dual 2-form to make it (and so the dual axion) massive. The gauge symmetry of the 3 -form constrains the form of these contributions in an advantageous way for many phenomenological applications. The description of the axions in terms of forms and their duals has also been key to the use of the weak gravity conjecture [2] to constrain transplanckian axion model building [3-20].

This formulation has been well understood:

- For the QCD axion, in [21-23], where the 3-form is actually the Chern-Simons composite 3 -form built out of the QCD gauge fields.

- In string compactifications producing axion monodromy [24, 25], as described in [26] connecting it to the earlier description in $[27,28] .^{1}$ In these cases, the 3 -form is a fundamental field, and its couplings arise from different sources, ranging from ChernSimons couplings to fluxes in the 10d action [26, 47] (see also [48]), torsion homology [26] (see also [49]) or topological brane-bulk couplings [43].

\footnotetext{
${ }^{1}$ For other works related to axion monodromy, see [15, 29-46].
} 
The two above phenomena, in particular the presence of fluxes and non-perturbative effects on D-brane gauge sectors, play an important role in several scenarios of moduli stabilization (and thus of their axion components), along the lines in [50]. Actually, the gauge non-perturbative effects can be described in string theory as particular cases of D-brane instanton effects wrapping the same cycle as the gauge D-branes in the compact space. In general, in string theory there are other non-perturbative effects from D-brane instantons not wrapping such cycles (sometimes dubbed stringy or exotic D-brane instantons [51-53], see $[54,55]$ for reviews), and contributing to the stabilization of axions as well. It is therefore natural to wonder about the 3-form description of these latter effects. Interestingly, there is no known description of this kind: since there is no gauge group associated to the cycles, we cannot use any composite Chern-Simons 3-form; on the other hand, for e.g. a D3-brane instanton on a 4-cycle, there is not any obvious corresponding harmonic form able to produce a 3 -form in the $4 \mathrm{~d}$ theory upon compactification.

In this paper we solve this question and provide the 3-form description for the stabilization of an axion by non-gauge D-brane instanton effects. The key idea is to notice that the stabilization occurs when the non-perturbative effect is included in the theory, so it is only then that we can hope to find a suitable 3 -form. Therefore, the internal form supporting the $4 \mathrm{~d} 3$-form must arise only in the geometry backreacted by the presence of the D-brane instanton, in the sense discussed in $[56,57]$. In general, these correspond to generalized geometries, so the corresponding form need not be harmonic with respect to the underlying CY metric, rather it corresponds to (a piece of) a generalized calibration.

We study this in the particular example of D3-brane instantons on 4-cycles, but the lesson is general (as expected from T-duality / mirror symmetry). Also, we show that the picture is compatible with D-brane instantons corresponding to gauge nonperturbative effects.

The paper is organized as follows. In section 2 we review the 3 -form description of axion stabilization and its interplay with axion monodromy and non-perturbative gauge dynamics. In section 3 we provide the 3 -form description of axion potentials induced by non-gauge D-brane instantons: after posing the question in section 3.1, we review the D3brane instanton backreacted geometry in section 3.2, and obtain the $4 \mathrm{~d} 3$-form and its couplings in section 3.3. A simple example is displayed in section 3.4. Section 3.5 describes the generalization, in particular the mirror picture of D2-brane instantons in type IIA compactifications. In section 4 we discuss the case of gauge D-brane instantons. Finally, section 5 contains our final remarks.

\section{Review of 3-forms and monodromy.}

Consider an axion $\phi$, regarded just as a scalar taking values in a circle (i.e. with discrete periodicity $^{2} 2 \pi$ ) and with an (approximate) shift symmetry. In many applications one is interested in generating a non-trivial potential for this axion, violating precisely this shift symmetry. For simplicity we consider the potential expanded at quadratic order around

\footnotetext{
${ }^{2}$ For simplicity we set the axion decay constant to $f_{\phi}=1$.
} 
a minimum, as for instance arises in moduli stabilization; the general picture is however more general. Hence we have the lagrangian

$$
|d \phi|^{2}+\mu^{2} \phi^{2}
$$

A potential of this kind is naively not compatible with the axion periodicity, but may be made so by including multivalued branches for the potential, thus inducing axion monodromy. The description of the periodicity properties are automatically implemented by using a dual formulation, in terms of a 3 -form $c_{3}$ eating up the $4 \mathrm{~d}$ dual 2 -form field $b_{2}$ (defined by $* d b_{2}=d \phi$, in $4 \mathrm{~d}$ ). This is described by the following lagrangian ${ }^{3}$

$$
\left|d b_{2}+n c_{3}\right|^{2}+\left|F_{4}\right|^{2}
$$

where $F_{4}=d c_{3}$. This theory has the gauge invariance

$$
c_{3} \rightarrow c_{3}+d \Lambda_{2} \quad, \quad b_{2} \rightarrow b_{2}-n \Lambda_{2}
$$

As emphasized in [26], it is this gauge symmetry that protects the flatness of the axion potential against uncontrolled corrections even in transplanckian field ranges. Dualizing the 2 -form back into the axion $\phi$, we obtain the Kaloper-Sorbo description of axion monodromy $[27,28,35,59]$

$$
|d \phi|^{2}+n \phi F_{4}+\left|F_{4}\right|^{2}
$$

Integrating out the non-dynamical 4 -form field strength $F_{4}$ one recovers a potential with the structure (2.1).

The idea can be generalized to other potentials by considering corrections, which due to the gauge invariance, (2.3) must be in terms of higher powers in $F_{4}$. This leads for instance to the flattening in [60].

At this point we would like to emphasize an important clarification: the description of the massive axion in terms of a 3 -form eating up the dual 2-form, or in terms of a coupling between (a function of) the axion and the 4-form field strength, is not necessarily a signature of axion monodromy, but rather of the existence of a non-trivial axion potential. Axion monodromy arises in cases when the potential does not naively satisfy the axion periodicity. On the other hand, the 3 -form description should also hold for non-monodromic potentials (i.e. single-valued and consistent with the axion periodicity). Our main interest in this note is indeed the 3-form description of single instanton non-perturbative potentials, which indeed are periodic in the axion.

Before entering this discussion, we conclude this review with a brief recap of the 3 -form description of axion potentials induced by non-perturbative gauge dynamics. This has been studied in the context of QCD axions in [21-23], but we recast it for pure $\mathcal{N}=1 \mathrm{SU}(n)$ SYM, which will be useful later on. The axion belongs to a chiral multiplet $\phi=\operatorname{Im} T$, and we have a coupling

$$
S_{\mathrm{SYM}}=\int d^{2} \theta d^{4} x T W_{\alpha} W^{\alpha} \sim \int_{4 d} \phi \operatorname{tr} F^{2}+\ldots
$$

\footnotetext{
${ }^{3}$ We allow for a $\mathbf{Z}_{n}$ discrete gauge symmetry, see $[26,48,58]$ for discussions of such system and the corresponding $\mathbf{Z}_{n}$ charged domain walls.
} 
The non-perturbative dynamics produces a gaugino condensate superpotential

$$
W=\omega^{k} \Lambda^{3}=\omega^{k} e^{-\frac{T}{n}}
$$

where $\omega=e^{2 \pi i / n}$ and $\Lambda=\exp \left(-\frac{T}{3 n}\right)$ is the SYM dynamical scale, regarded as a function of $T$.

The theory has $n$ gapped vacua, which differ by the value of $\langle\phi\rangle$ (the phase of the gaugino condensate), in the sense that a shift $\phi \rightarrow \phi+2 \pi$ changes the $k^{\text {th }}$ vacuum to the $(k+1)^{t h}$. Therefore the potential has a periodicity of $\phi \sim \phi+2 \pi n$. On the other hand, the axion should actually have a periodicity of $2 \pi$; in fact, this is ensured because there is a 3 -form description of the above axion potential. This follows from realizing that the coupling (2.5) describes a coupling to a 4 -form $\phi F_{4}$ structure, with $F_{4}=\operatorname{tr} F^{2}$. Namely, the 3 -form is the composite Chern-Simons 3 -form $c_{3}=\omega_{3}^{\mathrm{CS}}$ (defined by $d \omega_{3}^{\mathrm{CS}}=\operatorname{tr} F^{2}$ ).

In other words, although the potential is periodic with a period $2 \pi n$, the periodicity of $2 \pi$ is achieved via a monodromic structure, but with a finite number $n$ of branches. This is clearly associated with the $\mathbf{Z}_{n}$ discrete symmetry among the $n$ vacua of $\mathcal{N}=1$ SYM. The structure of domain walls among vacua is naturally described in terms of the (composite) 3 -form description [21-23].

\section{3-forms from D-brane instanton backreaction}

\subsection{The puzzle}

In string theory, there are non-perturbative contributions to the superpotential beyond the above non-perturbative gauge dynamics contributions. These come from, for instance, euclidean D-brane instantons which do not correspond to gauge theory instantons. A prototypical example is provided by D3-branes instantons wrapped on 4-cycles in CY compactifications, ${ }^{4}$ such as those used in moduli stabilization in [50]. In our discussions we will assume the instantons to indeed produce superpotential terms; it would be interesting, but beyond our scope, to develop an understanding of a dual description for instanton effects generating higher F-terms due to extra fermion zero modes [61, 62]. Denoting by $T$ the complex modulus associated to the wrapped 4-cycle, we have a superpotential

$$
W_{\mathrm{np}}=A e^{-T}
$$

where $A$ is a prefactor depending on complex structure moduli (and possibly also on open string moduli), whose detailed structure is not essential for our present purposes.

We expect the axion potential induced by these instantons to admit a description in terms of a 3-form eating up the dual 2-form. On the other hand, there is no obvious candidate for such a 3 -form in the $4 \mathrm{~d}$ CY compactification: the only available RR fields are even-degree gauge potentials, so the 3 -form should arise from the RR 6-form integrated over a 3-cycle. However, there is no natural pairing between a 3-cycle and a 4-cycle in a CY so as to support the topological coupling $\phi F_{4}$ ultimately responsible for the axion

\footnotetext{
${ }^{4}$ In general, these may carry world-volume fluxes, but for simplicity we will restrict to the case of trivial gauge backgrounds.
} 
potential. Therefore there is no natural candidate for the 3 -form coupling to the axion to reproduce its potential.

The solution to this problem is to follow the intuition gained in the discussion of the SYM superpotential. In that case, the 3-form arises only when the existence of nonperturbative sectors in the gauge theory are taken into account; namely, the fact that $F_{4}=\operatorname{tr} F^{2}$ implies that the presence of the instantons is built-in in the coupling $\phi F_{4}$. The implementation of a similar concept for non-gauge D-brane instantons requires proposing that the 3-form describing the axion stabilization should be looked for not in the original CY geometry, but rather in the geometry perturbed by the gravitational backreaction of the D-brane instantons. This perturbation of the geometry has been studied in the literature in $[56,57]$ by exploiting the technology of generalized geometry.

\subsection{D-brane instanton backreaction}

In this section we review ideas in $[56,57]$ to describe the backreaction of D-brane instantons in terms of generalized geometry. The uninterested reader may wish to jump to section 3.3.

The effect of D-brane instantons can be encoded in the underlying CY geometry by means of a deformation turning the $\mathrm{SU}(3)$ holonomy into (in general) an $\mathrm{SU}(3) \times \mathrm{SU}(3)$ structure, associated to the existence of two spinors (not covariantly constant due to the deformation) corresponding to a $4 \mathrm{~d} \mathcal{N}=1$ supersymmetry (possibly in AdS). Focusing already in the type IIB case, the two spinors are written

$$
\epsilon_{1}=\zeta_{+} \otimes \eta_{+}^{(1)}+\zeta_{-} \otimes \eta_{-}^{(1)} \quad, \quad \epsilon_{2}=\zeta_{+} \otimes \eta_{+}^{(2)}+\zeta_{-} \otimes \eta_{-}^{(2)}
$$

here $\zeta_{+}$and $\eta_{+}$are complex conjugate of $\zeta_{-}, \eta_{-}$, and $\zeta_{+}$is the $4 \mathrm{~d}$ spinor specifying the $\mathcal{N}=1$ supersymmetry, and satisfying $\nabla_{\mu} \zeta_{-}=-\frac{1}{2} W_{0} \gamma_{\mu} \zeta_{+}$, where $W_{0}$ is the superpotential at the AdS minimum (and for the Minkowski case we just have $W_{0}=0$ ).

The spinors $\eta^{(1,2)}$ can be used to define two polyforms,

$$
\Psi_{ \pm}=-\frac{i}{\left\|\eta^{(1)}\right\|^{2}} \sum_{l} \frac{1}{l !} \eta_{ \pm}^{(2) \dagger} \gamma_{m_{1} \ldots m_{l}} \eta_{+}^{(1)} d y^{m_{l}} \wedge \ldots \wedge d y^{m_{1}}
$$

Noticing the chirality of the spinors in the sandwich, the polyform $\Psi_{+}$contains even degree forms and $\Psi_{-}$contains odd degree forms. A common alternative notation is (for type IIB) $\Psi_{1}=\Psi_{+}$and $\Psi_{2}=\Psi_{-}$.

The familiar case of $\mathrm{SU}(3)$ structure corresponds to $\eta^{(2)} \sim \eta^{(1)}$ and leads to $\Psi_{+} \sim e^{i J}$ and $\Psi_{-} \sim \Omega$. For SU(3) holonomy the spinors are covariantly constant and the polyforms are closed.

The compactification ansatz is

$$
d s^{2}=e^{2 A(y)} g_{\mu \nu}(x) d x^{\mu} d x^{\nu}+h_{m n}(y) d y^{m} d y^{n}
$$

The 10d fields can be organized in complex quantities, in agreement with the $4 \mathrm{~d}$ susy structure. One holomorphic quantity is

$$
\mathcal{Z} \equiv e^{3 A-\Phi} \Psi_{2}
$$


where $\Phi$ is the $10 \mathrm{~d}$ dilaton (not to be confused with the $4 \mathrm{~d}$ axion). This is motivated because it provides the calibration for BPS domain wall D-branes (notice that in IIB, $\Psi_{2}$ calibrates odd-dimensional cycles). In other words, the tension of a D-brane BPS domain wall is obtained by integrating the above form over the wrapped cycle. For instance, for standard CY compactifications, a D5-brane on a supersymmetric 3-cycle $\Pi$ provides a $4 \mathrm{~d}$ BPS domain wall, whose tension is given by $\int_{\Pi} \mathcal{Z}_{(3)} \sim \int_{\Pi} \Omega$, where the subindex (3) denotes the 3-form part of the polyform

The second quantity is

$$
\mathcal{T} \equiv e^{-\Phi} \operatorname{Re} \Psi_{1}+i \Delta C
$$

where $\Delta C$ describes the RR backgrounds not encoded in the background fluxes $\bar{F}$, i.e. the RR fluxes are split as $F=\bar{F}+d \Delta C$. The above complexification is motivated by the generalized calibration of BPS D-brane instantons (notice that in IIB, $\Psi_{1}$ calibrates even-dimensional cycles). For instance, for standard CY compactifications, a D3-brane wrapped on a holomorphic 4-cycle $\Sigma$ provides a 4 d BPS instanton whose action is given by $\int_{\Sigma} \mathcal{T}_{(4)} \sim \int_{\Sigma} e^{i J} \sim \int_{\Sigma} J \wedge J$.

\subsection{The 3-form and its coupling}

From the supersymmetry conditions recast in terms of the $10 \mathrm{~d}$ version of the $4 \mathrm{~d}$ fields $\mathcal{Z}$, $\mathcal{T}$, one can show that, in a weak coupling expansion, we have

$$
d_{H} \mathcal{Z}=\frac{2 i}{n} W_{\mathrm{np}} \delta_{2}(\Sigma)
$$

where $d_{H}=d+H \wedge$, so the 2-form term above is just $d_{H} \mathcal{Z}_{(1)}=d \mathcal{Z}_{(1)}$. This encodes the backreaction of the instanton effect on the $10 \mathrm{~d}$ geometry in terms of the appearance of a 1-form component $\mathcal{Z}_{(1)}$ of $\mathcal{Z}$, which was absent in the CY geometry.

This equation defines an special 1 -form $\alpha_{1} \equiv \mathcal{Z}_{(1)}$. It is associated to the globally defined supersymmetric spinor, in the presence of the non-perturbative correction to the geometry. Its structure can be obtained by integrating the above equation. For the particular case of a D3-brane instanton $(n=1)$ on a holomorphic 4-cycle defined by the equation $f=0$, one obtains [56]

$$
\mathcal{Z}_{(1)} \sim d f \tilde{W}_{\mathrm{np}}
$$

where the tilded superpotential has the dependence on open string degrees of freedom removed.

One intuitive way to understand the above expression is to notice that, in a theory containing gauge D3-branes (i.e. D3-branes sitting at a point in the internal space), the $4 \mathrm{~d}$ superpotential as a function of the D3-brane position is obtained by considering a 1chain $L$ joining two different points in the $\mathrm{CY}$ and integrating $\mathcal{Z}_{(1)}$ over it. This follows because $\mathcal{Z}_{(1)}$ is the calibrating form for a D3-brane wrapped on the 1-chain $L$, which defines a domain wall interpolating between the two configurations of the D3-branes at the two (end)points. We thus have

$$
\Delta W \sim \int_{L} \mathcal{Z}_{(1)}=f \tilde{W}_{\mathrm{np}}
$$


where we regard $f$ as the D3-brane position in the direction normal to the instanton 4cycle. The result therefore reproduces the familiar dependence on open string moduli, microscopically associated to Ganor strings [63] (see also [64]).

In the following we recast (3.7) as $d \alpha_{1}=\beta_{2}$, and we use both $\alpha_{1}$ and $\beta_{2}$ as internal profiles for the KK reduction of higher-dimensional form fields in the backreacted geometry. This is similar to the non-harmonic forms used in KK reduction of massive U(1)'s, and studied in [49] in compactification spaces with torsion (co)homology.

We may use these forms to perform the KK reduction of the $10 \mathrm{~d}$ RR 4 -form $C_{4}$ as

$$
C_{4}=\alpha_{1}(y) \wedge c_{3}(x)+\beta_{2}(y) \wedge b_{2}(x)+\ldots
$$

This produces a 3 -form in $4 \mathrm{~d}$ spacetime, naturally associated to the non-perturbative effect, and a 2-form, dual of $\phi$. Moreover, is it clear that the 3-form is eating up the 2-form, by noticing that the field strength $F_{5}$ has a term

$$
F_{5}=\left(1+*_{10 d}\right)\left(\beta_{2} \wedge\left(c_{3}+d b_{2}\right)-\alpha_{1} \wedge F_{4}\right)
$$

where $*_{10 d}$ is added to take the self-duality of $F_{5}$ into account. This clearly has the gauge invariance

$$
c_{3} \rightarrow c_{3}+d \Lambda_{2} \quad, \quad b_{2} \rightarrow b_{2}-\Lambda_{2}
$$

This implies that the 3 -form is eating up the 2 -form to become massive, and correspondingly provides a dual description of the axion $\phi$ becoming massive, as in section 2 .

It is also straightforward to show that this 3-form has a Kaloper-Sorbo coupling to the axion. We focus just on the leading term $\phi F_{4}$, where $\phi=\int_{\Sigma} C_{4}$, and $F_{4}=d c_{3}$. We simply massage the kinetic term of the (self-dual) 4 -form and focus on the components in (3.11) as follows

$$
\int_{10 d} F_{5} \wedge * F_{5}=-\int_{10 d} C_{4} \wedge d * F_{5} \rightarrow \int_{10 d} C_{4} \wedge \beta_{2} \wedge F_{4}=\int_{4 d} \phi F_{4}
$$

where we used $\beta_{2} \sim \delta_{2}(\Sigma)$.

\subsection{Some toroidal examples}

To flesh out this somewhat abstract description, let us now consider a toroidal compactification $M_{4} \times \mathbf{T}^{6}$, where for simplicity we take a factorizable, $\mathbf{T}^{6}=\mathbf{T}^{2} \times \mathbf{T}^{2} \times \mathbf{T}^{2}$ with local complex coordinates be $z_{1}, z_{2}, z_{3}$. Let us study the backreaction caused by a instantonic D3-brane wrapping the 4 -cycle $\Sigma_{4}$ defined by $z_{3}=0$. Using the general formulas in $[56,65]$, the complex structure $\mathcal{Z}=\Omega$ gets corrected, becoming a generalized complex structure with a 1 -form piece

$$
Z_{(1)} \sim e^{-T} d z_{3}
$$

This is the 1-form to be used to produce the $4 \mathrm{~d} 3$-form upon compactification of the $10 \mathrm{~d}$ RR 4-form.

Notice that it actually corresponds to $d z_{3}$, a harmonic 1-form already present in the underlying toroidal geometry. Therefore there seems to be essentially no new geometric 
structure associated to the backreacted geometry, namely, no axion potential due to the instanton effect. This feature is clearly related to the existence of extra harmonic forms in the $\mathbf{T}^{6}$ geometry, which are not present in generic CYs. However, it nicely dovetails the expectation that D3-brane instantons in toroidal geometries have additional fermion zero modes, and do not produce non-perturbative superpotentials for the corresponding moduli.

In order to actually get non-trivial structure, we can consider orbifolds which remove the extra harmonic forms, and produce genuine CY geometries. Consider for instance $\mathbf{T}^{6} /\left(\mathbf{Z}_{2} \times \mathbf{Z}_{2}\right)$, where the generators of the orbifold group act as $\theta:\left(z_{1}, z_{2}, z_{3}\right) \rightarrow$ $\left(-z_{1}, z_{2},-z_{3}\right)$, and $\omega:\left(z_{1}, z_{2}, z_{3}\right) \rightarrow\left(z_{1},-z_{2},-z_{3}\right)$. To describe the quotient, we introduce local coordinates ${ }^{5}$ by building orbifold invariants, $u_{i}=z_{i}^{2}, t=z_{1} z_{2} z_{3}$, subject to $u_{1} u_{2} u_{3}=t^{2}$. The instanton wrapped on the second and third torus is defined by $f \equiv u_{3}=0$, so $f=u_{3}$, and we have

$$
\mathcal{Z}_{(1)} \sim d u_{3} \sim z_{3} d z_{3}
$$

It is now clear that the 1 -form supporting the 3 -form in the compactification of the $10 \mathrm{~d}$ 4 -form is non-harmonic with respect to the original CY geometry.

\subsection{Generalization}

Although we have focused on the case of axions with potential arising from D3-brane instantons on 4-cycles, the ideas hold for general RR axions associated to other cycles, and with potentials arising from the corresponding wrapped D-brane instantons. In order to illustrate this, we consider the mirror configuration of type IIA with axions arising from the RR 3-form on a 3-cycle, stabilized by D2-brane instantons.

Let us thus study the mirror dual to the configuration of D3-branes on a 4-cycle, in the setup of a general CY (alternatively the main ideas can already be illustrated in the toroidal examples in section 3.4). Consider the CY in the large complex structure limit, where it can be regarded as a $\mathbf{T}^{3}$ (parametrized by coordinates $y_{i}, 1,2,3$, ) fibered over a $3 \mathrm{~d}$ base, with local coordinates $x_{i}, i=1,2,3$. The complex coordinates are locally $z_{i}=x_{i}+i y_{i}$, and we consider a holomorphic 4-cycle given locally by $z_{3}=0$, i.e. spanning $x_{1}, y_{1}, x_{2}, y_{2}$.

The mirror dual can be obtained by applying three T-dualities [66], along the coordinates $y_{i}$. The D3-brane instanton thus turns into a D2-brane wrapped on the 3-cycles locally spanned by $x_{1}, x_{2}, y_{3}^{\prime}$ (with the prime denoting the T-dual coordinate). One further sees that the complex structure deformation $Z_{(1)} \sim d f=d x_{3}+i d y_{3}$ gives rise to a polyform

$$
\delta \mathcal{T}=\mathcal{T}_{(2)}+\mathcal{T}_{(4)}
$$

This follows from the fact that $\mathcal{Z}_{(1)}$ is eventually use to expand the RR forms and obtain the $4 \mathrm{~d} 3$-form. Hence, these are the 2- and 4-form components of (3.6) produced by the backreaction of the D2-instanton, as we argue later on. Before that, let us conclude that the expansion of the RR polyform $\mathcal{C}=C_{1}+C_{3}+C_{5}+C_{7}$ along $\delta \mathcal{T}$ produces the $4 \mathrm{~d} 3$-form

\footnotetext{
${ }^{5} \mathrm{~A}$ global construction is easily produced by using Weierstrass equations for the 2-tori, but we will not need this extra complication.
} 
as follows

$$
\mathcal{C}=\delta \mathcal{T} \wedge c_{3} \rightarrow C_{7}=\mathcal{T}_{(4)} \wedge c_{3}, C_{5}=\mathcal{T}_{(2)} \wedge c_{3}
$$

where the $c_{3}$ in the last two expansions is understood to be the same $4 \mathrm{~d} 3$-form.

Let us finish by arguing further that the above $\delta \mathcal{T}$ is indeed the backreaction corresponding to the D2-brane instanton. In the original picture in section 3.3 we considered the superpotential in the theory in the presence of a gauge D3-brane, given by the integral of the calibrating form over a 1-chain. Under the mirror transformation we must consider the theory in the presence of a gauge D6-brane wrapped on the 3 -cycle $\Pi_{3}$ (i.e. the mirror $\mathbf{T}^{3}$ fiber, spanned by $\left.y_{i}^{\prime}, i=1,2,3\right)$. The superpotential is given by the integral of the calibrating form $\mathcal{T}$ over a (generalized) 4-chain $\Sigma$ interpolating between two (possibly generalized) 3-cycles (see e.g. [67])

$$
\Delta W_{D 6}=\int_{\Sigma} \delta \mathcal{T}
$$

We are interested in the superpotential as a function of one D6-brane complex modulus, given by one deformation of the special lagrangian 3-cycle, and the corresponding Wilson line along one 1-cycle in $\Pi_{3}$. The actual components turned on in this $\delta \mathcal{T}$ are $\mathcal{T}_{(4)}$, which will be integrated along the 4-chain produced by the deformation of the 3 -cycle $\Pi_{3}$, and a component $\mathcal{T}_{2}$ integrated over the 2-chain spanned by the 1-cycle in such deformation. The latter accounts for the contribution to the superpotential of the induced D4-brane charge arising from possible D6-brane worldvolume fluxes on the 2-forms Poincaré dual to the 1-cycle in $\Pi_{3}$.

The resulting variation of the superpotential is

$$
\Delta W_{D 6}=\int_{\Sigma_{4}} \mathcal{T}_{(4)}+\int_{\Sigma_{4}} \mathcal{T}_{(2)} \wedge \mathcal{F}=\int_{\Sigma_{4}} \mathcal{T}_{(4)}+\int_{\Sigma_{2}} \mathcal{T}_{(2)},
$$

where $\mathcal{F}$ is the magnetic field induced in the D6-brane.

Therefore, the only way to reproduce the D6-brane open string moduli dependence described by the Ganor zeroes is that the instanton backreaction indeed produces the deformation $\delta \mathcal{T}$ with components $\mathcal{T}_{(2)}$ and $\mathcal{T}_{(4)}$.

\section{Gauge non-perturbative effects}

There are D3-brane instantons which admit the interpretation of gauge theory instantons. This happens when the D3-brane instanton wraps precisely the same 4-cycle as a stack of 4d spacetime-filling D7-branes.

The description of stabilization of axions coupling to non-abelian gauge interactions has been recast in terms of coupling to the composite Chern-Simons 3-form in [21-23] cf. section 2. It is natural to ask about any possible interplay between this and the 3 -form discussed in earlier sections.

Actually, the 3-form in earlier sections arises only when the D-brane instantons backreact, in other words when the gauge dynamics is geometrized. This implies that we must 
consider the geometry that results when the D7-branes, together with the euclidean D3brane instantons, are backreacted on the geometry. The resulting configuration no longer contains open string degrees of freedom, as everything is encoded in the backreacted geometry. Therefore the relation between the 3 -forms is essentially holographic: on one side there are open string degrees of freedom, and the axion stabilization mechanism is described as in [21-23] in terms of a 3-form constructed out of the open string sector gauge fields; on the other side, there is a backreacted geometry, and no open string degrees of freedom, and the axion stabilization arises from a 3 -form supported by the distorted geometry.

To flesh out the latter claim, let us consider the description of the backreaction of D7-branes and their euclidean D3-brane instanton effects. We concentrate in the case $\mathcal{N}=1$ SYM, where the instantons contribute to the superpotential, and therefore to the axion stabilization.

To describe the backreacted geometry, we borrow results from [65]. The backreaction of the D7-branes, at the perturbative level (i.e not including the euclidean D3-brane instantons) is defined by

$$
\mathcal{Z}=\Omega \quad, \quad \mathcal{T}=e^{-\Phi} \exp \left(i e^{\Phi / 2} J\right)
$$

(where here $\Phi$ is the dilaton).

To this order there is no deformation of $\mathcal{Z}$, and moreover no 1-form that can support the 3 -form. The latter is however generated when the non-perturbative gaugino condensate (which is described by (fractional) euclidean D3-branes) is included. The gaugino condensate $\langle S\rangle=\langle\lambda \lambda\rangle=e^{2 \pi i k / n} \Lambda^{3}$ is a non-zero vev for the gaugino bilinear. In [65] it was found that

$$
d \mathcal{Z}=i \ell_{s}^{4}\langle S\rangle \delta_{2}(\Sigma)
$$

This is exactly as in (3.7), thereby confirming the anticipation that the backreacted D7/D3 system can support a 3 -form in agreement with the mechanism in earlier sections.

\section{Conclusions}

In this note we have provided the description of the axion potential from non-gauge D-brane instanton effects in terms of a 3 -form eating up the 2 -form dual to the axion. The 3 -form arises from the KK reduction of higher-dimensional RR fields in the generalized geometry arising when the D-brane instanton backreaction is taken into account. The mechanism also holds for D-brane instantons corresponding to gauge instantons, in which case the generalized geometry description of the axion couplings can be regarded as holographically related to earlier 3-form descriptions of stabilization of QCD-like axions.

Our works puts axion potentials from non-perturbative effects in a similar footing to other stabilization mechanisms, like flux compactifications. We hope this can improve the study of the interplay between different stabilization mechanisms in string theory. In another line, given recent results in applying the Weak Gravity Conjecture to axion models in terms of their dual 3-forms [15], we expect our analysis to allow similar analysis for nonperturbative axion potentials from instantons.

We hope to come back to these questions in the near future. 


\section{Acknowledgments}

We would like to thank L. Ibáñez, F. Marchesano, A. Retolaza and G. Zoccarato for useful discussions. E. G. and A. U. are partially supported by the grants FPA2015-65480$P$ from the MINECO, the ERC Advanced Grant SPLE under contract ERC-2012-ADG20120216-320421 and the grant SEV-2012-0249 of the "Centro de Excelencia Severo Ochoa" Programme.

Open Access. This article is distributed under the terms of the Creative Commons Attribution License (CC-BY 4.0), which permits any use, distribution and reproduction in any medium, provided the original author(s) and source are credited.

\section{References}

[1] R.D. Peccei and H.R. Quinn, CP conservation in the presence of instantons, Phys. Rev. Lett. 38 (1977) 1440 [INSPIRE].

[2] N. Arkani-Hamed, L. Motl, A. Nicolis and C. Vafa, The string landscape, black holes and gravity as the weakest force, JHEP 06 (2007) 060 [hep-th/0601001] [INSPIRE].

[3] A. de la Fuente, P. Saraswat and R. Sundrum, Natural inflation and quantum gravity, Phys. Rev. Lett. 114 (2015) 151303 [arXiv:1412.3457] [INSPIRE].

[4] T. Rudelius, Constraints on axion inflation from the weak gravity conjecture, JCAP 09 (2015) 020 [arXiv: 1503.00795] [INSPIRE].

[5] M. Montero, A.M. Uranga and I. Valenzuela, Transplanckian axions!?, JHEP 08 (2015) 032 [arXiv:1503.03886] [INSPIRE].

[6] J. Brown, W. Cottrell, G. Shiu and P. Soler, Fencing in the swampland: quantum gravity constraints on large field inflation, JHEP 10 (2015) 023 [arXiv: 1503.04783] [INSPIRE].

[7] T.C. Bachlechner, C. Long and L. McAllister, Planckian axions and the weak gravity conjecture, JHEP 01 (2016) 091 [arXiv: 1503.07853] [INSPIRE].

[8] A. Hebecker, P. Mangat, F. Rompineve and L.T. Witkowski, Winding out of the swamp: evading the weak gravity conjecture with F-term winding inflation?, Phys. Lett. B 748 (2015) 455 [arXiv: 1503.07912] [INSPIRE].

[9] J. Brown, W. Cottrell, G. Shiu and P. Soler, On axionic field ranges, loopholes and the weak gravity conjecture, JHEP 04 (2016) 017 [arXiv: 1504.00659] [INSPIRE].

[10] D. Junghans, Large-field inflation with multiple axions and the weak gravity conjecture, JHEP 02 (2016) 128 [arXiv: 1504.03566] [INSPIRE].

[11] B. Heidenreich, M. Reece and T. Rudelius, Weak gravity strongly constrains large-field axion inflation, JHEP 12 (2015) 108 [arXiv: 1506.03447] [INSPIRE].

[12] S. Bielleman, L.E. Ibáñez and I. Valenzuela, Minkowski 3-forms, flux string vacua, axion stability and naturalness, JHEP 12 (2015) 119 [arXiv:1507.06793] [INSPIRE].

[13] E. Palti, On natural inflation and moduli stabilisation in string theory, JHEP 10 (2015) 188 [arXiv:1508.00009] [INSPIRE].

[14] B. Heidenreich, M. Reece and T. Rudelius, Sharpening the weak gravity conjecture with dimensional reduction, JHEP 02 (2016) 140 [arXiv: 1509.06374] [INSPIRE]. 
[15] L.E. Ibáñez, M. Montero, A. Uranga and I. Valenzuela, Relaxion monodromy and the weak gravity conjecture, JHEP 04 (2016) 020 [arXiv: 1512.00025] [INSPIRE].

[16] A. Hebecker, F. Rompineve and A. Westphal, Axion monodromy and the weak gravity conjecture, JHEP 04 (2016) 157 [arXiv: 1512.03768] [INSPIRE].

[17] A. Hebecker, J. Moritz, A. Westphal and L.T. Witkowski, Towards axion monodromy inflation with warped KK-modes, Phys. Lett. B 754 (2016) 328 [arXiv:1512.04463] [INSPIRE].

[18] J.P. Conlon and S. Krippendorf, Axion decay constants away from the lamppost, JHEP 04 (2016) 085 [arXiv: 1601.00647] [INSPIRE].

[19] F. Baume and E. Palti, Backreacted axion field ranges in string theory, JHEP 08 (2016) 043 [arXiv: 1602.06517] [INSPIRE].

[20] B. Heidenreich, M. Reece and T. Rudelius, Axion experiments to algebraic geometry: testing quantum gravity via the weak gravity conjecture, Int. J. Mod. Phys. D 25 (2016) 1643005 [arXiv: 1605.05311] [INSPIRE].

[21] G. Dvali, Three-form gauging of axion symmetries and gravity, hep-th/0507215 [INSPIRE].

[22] G. Dvali, R. Jackiw and S.-Y. Pi, Topological mass generation in four dimensions, Phys. Rev. Lett. 96 (2006) 081602 [hep-th/0511175] [inSPIRE].

[23] G. Dvali, S. Folkerts and A. Franca, How neutrino protects the axion, Phys. Rev. D 89 (2014) 105025 [arXiv: 1312.7273] [INSPIRE].

[24] E. Silverstein and A. Westphal, Monodromy in the CMB: gravity waves and string inflation, Phys. Rev. D 78 (2008) 106003 [arXiv:0803.3085] [INSPIRE].

[25] L. McAllister, E. Silverstein and A. Westphal, Gravity waves and linear inflation from axion monodromy, Phys. Rev. D 82 (2010) 046003 [arXiv: 0808.0706] [INSPIRE].

[26] F. Marchesano, G. Shiu and A.M. Uranga, F-term axion monodromy inflation, JHEP 09 (2014) 184 [arXiv: 1404.3040] [INSPIRE].

[27] N. Kaloper and L. Sorbo, A natural framework for chaotic inflation, Phys. Rev. Lett. 102 (2009) 121301 [arXiv:0811.1989] [INSPIRE].

[28] N. Kaloper, A. Lawrence and L. Sorbo, An ignoble approach to large field inflation, JCAP 03 (2011) 023 [arXiv: 1101.0026] [inSPIRE].

[29] M. Berg, E. Pajer and S. Sjors, Dante's Inferno, Phys. Rev. D 81 (2010) 103535 [arXiv:0912.1341] [INSPIRE].

[30] L.E. Ibáñez and I. Valenzuela, BICEP2, the Higgs mass and the SUSY-breaking scale, Phys. Lett. B 734 (2014) 354 [arXiv: 1403.6081] [INSPIRE].

[31] E. Palti and T. Weigand, Towards large $r$ from $[p, q]$-inflation, JHEP 04 (2014) 155 [arXiv: 1403.7507] [INSPIRE].

[32] R. Blumenhagen and E. Plauschinn, Towards universal axion inflation and reheating in string theory, Phys. Lett. B 736 (2014) 482 [arXiv:1404.3542] [INSPIRE].

[33] L.E. Ibáñez and I. Valenzuela, The inflaton as an MSSM Higgs and open string modulus monodromy inflation, Phys. Lett. B 736 (2014) 226 [arXiv:1404.5235] [INSPIRE].

[34] A. Hebecker, S.C. Kraus and L.T. Witkowski, D7-brane chaotic inflation, Phys. Lett. B 737 (2014) 16 [arXiv: 1404.3711] [INSPIRE]. 
[35] N. Kaloper and A. Lawrence, Natural chaotic inflation and ultraviolet sensitivity, Phys. Rev. D 90 (2014) 023506 [arXiv: 1404.2912] [INSPIRE].

[36] M. Arends et al., D7-brane moduli space in axion monodromy and fluxbrane inflation, Fortsch. Phys. 62 (2014) 647 [arXiv: 1405.0283] [INSPIRE].

[37] S. Franco, D. Galloni, A. Retolaza and A. Uranga, On axion monodromy inflation in warped throats, JHEP 02 (2015) 086 [arXiv: 1405.7044] [INSPIRE].

[38] R. Blumenhagen, D. Herschmann and E. Plauschinn, The challenge of realizing F-term axion monodromy inflation in string theory, JHEP 01 (2015) 007 [arXiv:1409.7075] [INSPIRE].

[39] A. Hebecker, P. Mangat, F. Rompineve and L.T. Witkowski, Tuning and backreaction in F-term axion monodromy inflation, Nucl. Phys. B 894 (2015) 456 [arXiv:1411.2032] [INSPIRE].

[40] L.E. Ibáñez, F. Marchesano and I. Valenzuela, Higgs-otic inflation and string theory, JHEP 01 (2015) 128 [arXiv:1411.5380] [INSPIRE].

[41] I. García-Etxebarria, T.W. Grimm and I. Valenzuela, Special points of inflation in flux compactifications, Nucl. Phys. B 899 (2015) 414 [arXiv:1412.5537] [INSPIRE].

[42] R. Blumenhagen et al., A flux-scaling scenario for high-scale moduli stabilization in string theory, Nucl. Phys. B 897 (2015) 500 [arXiv:1503.07634] [INSPIRE].

[43] A. Retolaza, A.M. Uranga and A. Westphal, Bifid throats for axion monodromy inflation, JHEP 07 (2015) 099 [arXiv: 1504.02103] [INSPIRE].

[44] D. Escobar, A. Landete, F. Marchesano and D. Regalado, Large field inflation from D-branes, Phys. Rev. D 93 (2016) 081301 [arXiv:1505.07871] [InSPIRE].

[45] S. Bielleman, L.E. Ibáñez, F.G. Pedro and I. Valenzuela, Multifield dynamics in Higgs-otic inflation, JHEP 01 (2016) 128 [arXiv:1505.00221] [INSPIRE].

[46] R. Blumenhagen, C. Damian, A. Font, D. Herschmann and R. Sun, The flux-scaling scenario: de Sitter uplift and axion inflation, Fortsch. Phys. 64 (2016) 536 [arXiv: 1510.01522] [INSPIRE].

[47] L. McAllister, E. Silverstein, A. Westphal and T. Wrase, The powers of monodromy, JHEP 09 (2014) 123 [arXiv: 1405.3652] [INSPIRE].

[48] M. Berasaluce-Gonzalez, P.G. Camara, F. Marchesano and A.M. Uranga, Zp charged branes in flux compactifications, JHEP 04 (2013) 138 [arXiv:1211.5317] [INSPIRE].

[49] P.G. Camara, L.E. Ibáñez and F. Marchesano, RR photons, JHEP 09 (2011) 110 [arXiv:1106.0060] [INSPIRE].

[50] S. Kachru, R. Kallosh, A.D. Linde and S.P. Trivedi, de Sitter vacua in string theory, Phys. Rev. D 68 (2003) 046005 [hep-th/0301240] [INSPIRE].

[51] R. Blumenhagen, M. Cvetič and T. Weigand, Spacetime instanton corrections in $4 D$ string vacua: the seesaw mechanism for D-brane models, Nucl. Phys. B 771 (2007) 113 [hep-th/0609191] [INSPIRE].

[52] L.E. Ibáñez and A.M. Uranga, Neutrino Majorana masses from string theory instanton effects, JHEP 03 (2007) 052 [hep-th/0609213] [INSPIRE].

[53] B. Florea, S. Kachru, J. McGreevy and N. Saulina, Stringy instantons and quiver gauge theories, JHEP 05 (2007) 024 [hep-th/0610003] [INSPIRE]. 
[54] R. Blumenhagen, M. Cvetič, S. Kachru and T. Weigand, D-brane instantons in Type II orientifolds, Ann. Rev. Nucl. Part. Sci. 59 (2009) 269 [arXiv:0902.3251] [InSPIRE].

[55] L.E. Ibanez and A.M. Uranga, String theory and particle physics: an introduction to string phenomenology, Cambridge University Press, Cambridge U.K. (2012).

[56] P. Koerber and L. Martucci, From ten to four and back again: how to generalize the geometry, JHEP 08 (2007) 059 [arXiv:0707.1038] [INSPIRE].

[57] P. Koerber and L. Martucci, Warped generalized geometry compactifications, effective theories and non-perturbative effects, Fortsch. Phys. 56 (2008) 862 [arXiv:0803.3149] [INSPIRE].

[58] M. Berasaluce-González, G. Ramírez and A.M. Uranga, Antisymmetric tensor $Z_{p}$ gauge symmetries in field theory and string theory, JHEP 01 (2014) 059 [arXiv:1310.5582] [INSPIRE].

[59] S. Dubovsky, A. Lawrence and M.M. Roberts, Axion monodromy in a model of holographic gluodynamics, JHEP 02 (2012) 053 [arXiv:1105.3740] [INSPIRE].

[60] X. Dong, B. Horn, E. Silverstein and A. Westphal, Simple exercises to flatten your potential, Phys. Rev. D 84 (2011) 026011 [arXiv: 1011.4521] [INSPIRE].

[61] C. Beasley and E. Witten, New instanton effects in supersymmetric QCD, JHEP 01 (2005) 056 [hep-th/0409149] [INSPIRE].

[62] I. Garcia-Etxebarria, F. Marchesano and A.M. Uranga, Non-perturbative F-terms across lines of BPS stability, JHEP 07 (2008) 028 [arXiv: 0805.0713] [INSPIRE].

[63] O.J. Ganor, A note on zeros of superpotentials in F-theory, Nucl. Phys. B 499 (1997) 55 [hep-th/9612077] [INSPIRE].

[64] D. Baumann et al., On D3-brane potentials in compactifications with fluxes and wrapped D-branes, JHEP 11 (2006) 031 [hep-th/0607050] [INSPIRE].

[65] A. Dymarsky and L. Martucci, D-brane non-perturbative effects and geometric deformations, JHEP 04 (2011) 061 [arXiv: 1012.4018] [INSPIRE].

[66] A. Strominger, S.-T. Yau and E. Zaslow, Mirror symmetry is T duality, Nucl. Phys. B 479 (1996) 243 [hep-th/9606040] [INSPIRE].

[67] F. Marchesano, D. Regalado and G. Zoccarato, On D-brane moduli stabilisation, JHEP 11 (2014) 097 [arXiv: 1410.0209] [INSPIRE]. 